\title{
Global epidemiology of dengue hemorrhagic fever: an update
}

\begin{abstract}
Dengue Hemorrhagic Fever (DHF) is a severe form of dengue fever (DF), which can be life threatening. The first recorded epidemic of dengue was reported in the late 18th century and affected Asia, Africa, and North America. Female mosquitoes, predominantly of the species Aedes aegyptiand less commonly, Aedes albopictus, are responsible for the transmission of the dengue virus. As the most dangerous and widespread mosquito-borne viral infection, the dengue virus affects more than 100 countries worldwide. Increasing 30fold in the last 50 years, the social and economic effects of dengue are far reaching and of worldwide concern. The disease is widespread throughout the tropics with local variations in risk influenced by rainfall, temperature, and unplanned rapid urbanization. Upto 400 million people are infected each year. Currently, vaccines are still under development for DHF; therefore, prevention is of utmost importance in lessening the impact of dengue on a global scale. This review article summarizes the global epidemiology of Dengue Hemorrhagic fever by utilizing the most recent and available information on the topic.
\end{abstract}

Keywords: Dengue fever, Dengue hemorrhagic fever, Aedes aegypti, Aedes albopictus
Volume 5 Issue 6 - 2017

\author{
Adekunle Sanyaolu,' Chuku Okorie, ${ }^{2}$ \\ Olanrewaju Badaru,' Khadijat Adetona, ${ }^{3}$ \\ Miriam Ahmed, ${ }^{3}$ Odunayo Akanbi, ${ }^{3}$ Juanita \\ Foncham, ${ }^{3}$ Shana Kadavil, ${ }^{3}$ Lorena Likaj, ${ }^{3}$ \\ Sarah Miraaj Raza, ${ }^{3}$ Erika Pearce, ${ }^{3}$ Roberta \\ Sylvester, ${ }^{3}$ Elizabeth Elizabeth ${ }^{3}$ \\ 'Federal Ministry of Health, Nigeria \\ ${ }^{2}$ Essex County College, USA \\ ${ }^{3}$ Saint James School of Medicine, USA
}

Correspondence: Adekunle Sanyaolu, Federal Ministry of Health, Abuja, Nigeria, Email sanyakunle@gmail.com

Received: September 25, 2017 | Published: October 24, 2017

\section{Introduction}

Although dengue was once a periodic disease that caused longinterval epidemics, today it is considered the most important mosquitoborne viral disease in the world. ${ }^{1}$ Per year, approximately fifty to one hundred million cases of dengue fever are recorded worldwide. ${ }^{2}$ Half a million of these cases result in the most severe form of dengue fever (DF) known as dengue hemorrhagic fever (DHF). ${ }^{2}$ DHF is a disease that predominantly affects children under 15 years of age and is characterized by the sudden onset of fever, thrombocytopenia, and vascular leak syndrome. ${ }^{3}$ DHF occurs due to an infection by the dengue virus, which is part of the Flaviviridae family. ${ }^{4}$ The virus is icosahedra, enveloped, single-stranded, positive-sense RNA. ${ }^{5}$ The icosahedra core is forty to fifty nanometers in diameter and contains the $\mathrm{C}$ protein that encapsulates the viral genome. ${ }^{5}$ Two viral proteins, $\mathrm{M}$ and $\mathrm{E}$, which compose the lipid envelope, surround the core. ${ }^{5}$ The infection is caused by one of the four serotypes of the dengue virus (DENV): DENV-1, DENV-2, DENV-3, and DENV-4. ${ }^{4}$ Infection by one of the serotypes does not provide immunity against infection with the other serotypes. ${ }^{4}$

If someone who has been previously infected is infected again, he or she has an increased risk of developing dengue shock syndrome (DSS) and DHF, which results in the weakening and rupture of vasculature and internal bleeding. ${ }^{4}$ Non-neutralizing antibodies can enhance the uptake of the virus by cells that express Fc receptors, thus allowing for antibody-dependent enhancement in DHF. ${ }^{4}$ Various species of Aedesmosquitoes are vectors for the dengue virus and transmit the virus by biting a human host. ${ }^{3}$ Aedes aegypti; a small, black-and-white, tropical and subtropical mosquito found in Central America, Latin America, Southeast Asia, and the Western Pacific, is the principal vector. ${ }^{3}$

\section{Epidemiology}

Today, DHF is regarded internationally as the most significant mosquito-borne viral disease. It is endemic to more than 100 countries worldwide, especially tropical and sub-tropical regions. ${ }^{6}$ In the United States, the DHF causing Aedes aegyptispecies can be found seasonally in Louisiana, southern Florida, New Mexico, Arizona, Texas, Georgia, Alabama, Mississippi, North and South Carolina, Kentucky, Oklahoma, and Tennessee. ${ }^{7}$ In the last 50 years, the incidence of dengue has increased 30 -fold. ${ }^{7}$

According to the World Health Organization (WHO), it is estimated that 50 to 100 million infections of dengue fever occur yearly. Of these cases 500,000 progress to DHF resulting in 22,000 deaths, mostly of children. ${ }^{1}$ Based on official data submitted to the WHO, cases of dengue across the Americas, South-East Asia and Western Pacific surpassed 1.2 million in 2008 and over 3 million in 2013. In 2013, 2.35 million cases of dengue were reported in the Americas alone, of which 37, 687 cases were of DHF. ${ }^{1}$ After the first known epidemic of DHF in 1953 to 1954 in the Philippines, the disease continued to spread throughout Southeast Asia. ${ }^{4}$

During the 1950s, 1960s, and most of the 1970s, dengue epidemic was rare in most of South and Central America because the primary mosquito vector, Aedes aegypti, was eradicated. However, once the eextermination was stopped, Aedes aegypti began to reinvade those regions. In 1997, Aedes aegyptiand the dengue virus gained a worldwide distribution again, ${ }^{3}$ (Figures 1,2).According to WHO,there are now over 2.5 billion people who live in areas where dengue/ DHF is endemic and are at risk for being infected with the dengue virus. ${ }^{1}$ Several factors contribute to the viral transmission by Aedes aegypti; including temperature, rainfall, rural-urban migration, population growth, stored water, increase solid waste which allows for larval habitats for the vector. ${ }^{8}$ There can also be outbreaks of travelassociated DHF. Dengue fever is the most widespread arbovirus infection worldwide. ${ }^{9}$

\section{Risk factors for DF and transmission of dengue virus}

Dengue fever is an important arthropod-borne viral disease. ${ }^{10}$ Research shows that the Aedes genus, primarily Aedes aegypti and Aedes albopictus mosquitoes, have adapted to living near areas of human habitation and are responsible for the transmission of dengue fever. The mosquitoes feed during the day and prefer human beings to other animals. ${ }^{11}$ Aedes aegyptiihas the highest human blood- 
feeding pattern at $76.9 \%$, followed by Aedes albopictus and Aedes vittatus mosquitoes, with $75 \%$ and $33.3 \%$ respectively. ${ }^{12}$ The incidence of DF has been associated with amount of vegetation, tree cover, housing quality, and surrounding land area. ${ }^{12}$

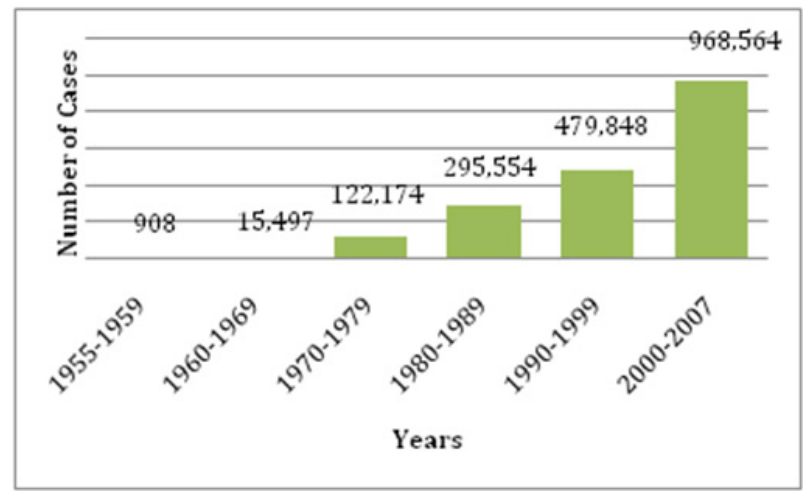

Figure I Average number of DF/DHF reported to WHO in the years provided. ${ }^{30}$

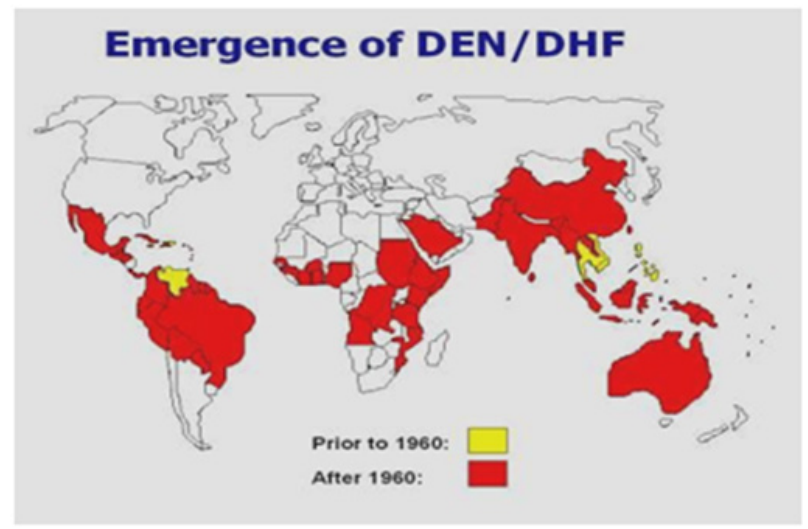

Figure 2 Distribution of dengue/ dengue hemorrhagic fever in the Americas, Caribbean, Africa and the Middle East.

Along with manmade breeding grounds, climate has also been shown to influence dengue ecology by affecting vector dynamics, agent development, and mosquito/human interaction.13 Temperature helps to influence vector development rates, mortality, behavior, and controls viral replication within the vector. Warm climates allow for larval development and an increased speed of virus replication, whereas cold climates slow down viral development and decrease the mosquitoes' survival time, thus decreasing the possibility of an infectious vector and decreasing the transmission of dengue fever. ${ }^{11}$ Temperature also interacts with rainfall as the chief regulator of evaporation, thereby directly affecting the availability of water habitats and indirectly affecting land cover and land use, which can promote or impede the growth of vector populations. ${ }^{13}$

Demographic and social changes such as population growth, urbanization, and modern transportation contribute greatly to the increase incidence and geographic spread of dengue activity. ${ }^{14}$ As travelers move from endemic areas, they tend to serve as vehicles for further spread. ${ }^{10}$ Viral strain virulence and genetic variation of the dengue virus also lend themselves to greater epidemic transmission potential of DHF. ${ }^{14}$

\section{Pathogenesis of dengue hemorrhagic fever}

As the mosquito feeds on the human host, it injects the dengue virus (DENV) into the bloodstream and surrounding epidermis and dermis. This result in the infection of immature Langerhans cells and keratinocytes through receptor-mediated endocytosis. ${ }^{15,16} \mathrm{E}$ protein, the surface protein, is reconfigured and allows for membrane fusion and release of the viral RNA into the infected cell. The non-structural proteins, NS1 and NS2A control viral RNA replication and NS1 may also be implicated in the activation of the complement cascade. ${ }^{16}$ The infected cells may then die via apoptosis or necrosis or migrate to the lymph nodes where macrophages are recruited, ${ }^{15}$ (Figure 3 ). Infection by any one of the four serotypes of dengue (DENV-1 through DENV4) could be asymptomatic in the majority of cases as the host immune system is able to neutralize it locally, but it may also cause a spectrum of clinical manifestations, the most severe form being DHF, which can progress to DSS and death. ${ }^{15} \mathrm{DHF}$ is clinically defined as a continuous fever of 2 to 7 days with mucosal or skin hemorrhagic manifestations associated with thrombocytopenia $\left(<100,000\right.$ cell $\left./ \mathrm{mm}^{3}\right)$ and a haematocrit greater than $20 \%$ above the baseline of the patient or same age population. ${ }^{17}$ The pathological hallmarks of DHF are plasma leakage from pleural or peritoneal surfaces and intrinsic Coagulopathy, which is clinically measured by either a positive tourniquet test, or by evidence of spontaneous bleeding such as petechiae or ecchymoses, gingival bleeding or epistaxis, or gastrointestinal hemorrhage. ${ }^{17,18}$

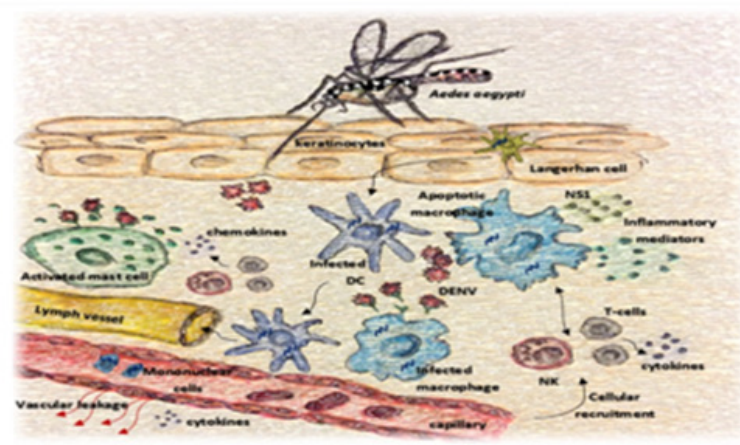

Figure 3 After being bitten by the carrier Aedes aegypti mosquito, immature Langerhans cells and keratinocytes are infected with DENV.The infected cells then move down into the lymph where macrophages are recruited. ${ }^{15}$ From here the infection is disseminated into various organs, including the liver, spleen and bone marrow. Necrosis of the bone marrow by DENV can lead to suppression of hematopoietic and decreased thrombogenesis in the blood..$^{18,19}$ The inflammatory response, caused by chemokine and cytokine release from mast cells, macrophages, and lymphocytes causes increased vascular permeability, platelet dysfunction, and thrombocytopenia and plasma leakage. ${ }^{18}$ Though the mechanism is not well understood this cascade also appears to cause spontaneous bleeding, leading to the clinical manifestations of petechiae, gingival bleeding, and gastrointestinal hemorrhage. ${ }^{18}$

This severe form of the disease often is a result of a secondary infection where the host is infected with a second serotype and a complex interaction between the virus and the immune response of the host ensues. ${ }^{19}$ Though the mechanism that causes this reaction is not well understood, evidence suggests that prior infection may create cross-reactive antibodies that bind the new serotype, but are unable to neutralize it. ${ }^{16,18}$ This is called the antibody-dependent enhancement (ADE) theory. ${ }^{16}$ It is thought that antibodies formed during previous infections against DENV structural proteins, such as E protein or $\mathrm{prM}$, are unable to neutralize the new pathogen and instead lead to enhanced binding to $\mathrm{F}_{c \gamma}$ receptor-positive cell enabling the amplified replication of the virus. ${ }^{19}$ In response, the immune system releases an exaggerated cytokine response, leading to increased vascular permeability and Coagulopathy. ${ }^{18}$

Mast cells have also been implicated in the pathogenesis of DHF. In some cases, mast cells appear to provide a protective function where anti-viral molecules are released by de granulation and other 
immune cells, such as T-lymphocytes and natural killer (NK) cells are recruited, all of which help to neutralize the virus..$^{20}$ However, in other cases, local control mechanisms cannot contain the virus, and it travels through the bloodstream or lymphatic system to activate mast cells in other organs such as the liver, spleen and lymph nodes. ${ }^{19}$ When de granulation occurs in these organs, proteases are released and inflammatory mediators, such as leukotrienes and vascular endothelial growth factor (VEGF), are synthesized, increasing the permeability of capillaries, and leading to plasma leakage. ${ }^{20}$ This is important as different groups of mast cells release distinct mediators during the de granulation process..$^{20}$ The virus-antibody complex, perhaps through the NS1 antigen of the dengue virus, and cytokine production also results in complement activation. The release of $\mathrm{C} 3 \mathrm{a}$ and $\mathrm{C} 5 \mathrm{a}$ fragments are known to increase vascular permeability and thus contribute to plasma leakage. ${ }^{18}$

The type and amount of cytokines released plays an important role in dictating the severity of disease. Macrophage inflammatory protein $1 \beta$ (MIP-1 $\beta$ ) appears to be an indicator of less severe disease as it is released by infected mononuclear cells and activated NK cells. It acts as a chemo attractant for more NK cells, luring them to the inflammatory site, limiting viral replication and the downstream cytokine effect. Interferon $\gamma$ (IFN- $\gamma$ ), on the other hand, has been shown to induce plasma leakage and to increase the uptake of dengue particles through increasing $\mathrm{Fc}$ receptors on target cells and is therefore associated with more severe clinical manifestations and poor prognosis. ${ }^{21}$ Other cytokines found to be increased in DHF that have been implicated in inducing plasma leakage include interleukin 2 (IL-2), tumor necrosis factor $\alpha$ (TNF- $\alpha$ ) macrophage migration inhibitory factor (MIF), monocyte chemo tactic protein-1 (MCP-1), interleukin-8 (IL-8), and high mobility group box-1 (HMGB-1). ${ }^{19}$ The effects of cytokine release is not limited to vascular permeability and plasma leakage, but is also implicated in the hemorrhagic manifestations of DHF. However, the underlying mechanism that causes bleeding in DHF is poorly understood. Though most infected cells are prone to death through apoptosis, some necrosis does occur resulting in the release of toxins, which activate the coagulation and fibrinolysis systems. When high levels of necrosis occur in the bone marrow due to high viral load, hematopoietic is suppressed, resulting in thrombolyticpenia. ${ }^{15}$ The resulting decrease in thrombogenesis and platelet dysfunction may cause capillary fragility and the resulting clinical manifestations seen in DHF. ${ }^{15}$ Some studies indicate that the involvement of the liver in DENV infection may be implicated in spontaneous bleeding tendencies, with a correlation between elevated liver enzyme levels and increased tendency for hemorrhage. ${ }^{15,22}$

\section{Clinical signs and symptoms}

The clinical manifestation of the disease includes asymptomatic infection, mild dengue fever (DF), dengue hemorrhagic fever (DHF), or dengue shock syndrome (DSS), that is often severe due to abnormal capillary permeability and plasma leakage. ${ }^{23}$ Severe dengue usually occurs among patients that have been infected previously with a dengue virus (i.e., secondary infection). High rates of severe dengue infection have also been reported in infants with primary dengue virus infection that are born by mothers who have been infected previously with a dengue virus. ${ }^{24}$

The initial phase of DHF appears as dengue fever. The symptomatology of this initial phase depends on the age of the patient and includes a sudden high fever (Sometimes with two 2 peaks-saddle backed), severe headache, decreased appetite, severe myalgia and arthralgia, a maculopapular skin rash which appears three to four days post the initial onset of the fever, retro-orbital pain, photophobia, lymphadenopathy, mild bleeding (epistaxis, gum bleeding, gastrointestinal bleeding, haematuria, and menorrhagia), and a positive tourniquet test. ${ }^{17} \mathrm{DHF}$ cases are characterized by four major clinical manifestations which are high fever, haemorrhagic phenomena, and often, hepatomegaly and circulatory failure. Acute febrile illness characteristic of the initial phase consists of temperatures greater than $39^{\circ} \mathrm{C}$ and remain so for 2-7days.

The aforementioned symptoms of initial phase progressively worsen with a continuous fever and headache, severe abdominal pain or tenderness, persistent vomiting, hematemesis, melena, petechiae, easy bruising, restlessness, lethargy, drowsiness, irritability, bleeding or bruising under the skin. ${ }^{17}$ After 2-7days of fever, a rapid fall in temperature is often accompanied by signs of circulatory disturbance of varying severity. Patient may sweat, be restless, and have cool extremeties and show changes in pulse rate and blood pressure. After 24-48 hours of the onset of DHF specific features, abnormal hemostasis and plasma leakage are observed to be the hallmark symptoms.

A good indicator of these advancements is a greater than $2 \mathrm{~cm}$ enlargement of the liver, since hepatomegaly tends to precedes plasma leakage. ${ }^{17,22}$ Many patient recover with or without fluid and electrolyte treatment. In severe cases, plasma loss can progress to shock and death if untreated. ${ }^{17}$ Research suggests that both a secondary infection of dengue and the exaggerated retaliation of the cytokine immune system response increase the predilection for a patient to develop DHF.

Viral products such as NS1 glycoprotein are believed to influence the complement activation and the vascular permeability resulting in plasma leakage that develops into ascites or pleural effusions. ${ }^{18}$ The cytokine response is also responsible for increasing the vascular permeability, as a consequence of which the intravascular oncotic pressure is reduced and facilitates the loss of fluid into the extra vascular compartments,$^{18}$ (Figure 4). The abnormal hemostasis consist of thrombocytopenia concurrent with an increase in haematocrit, ${ }^{17}$ prolonged partial thromboplastin time with reduced fibrinogen concentration, along with the previously mentioned petechiae, severe gastrointestinal hemorrhages, ecchymosis and epistaxis..$^{18}$

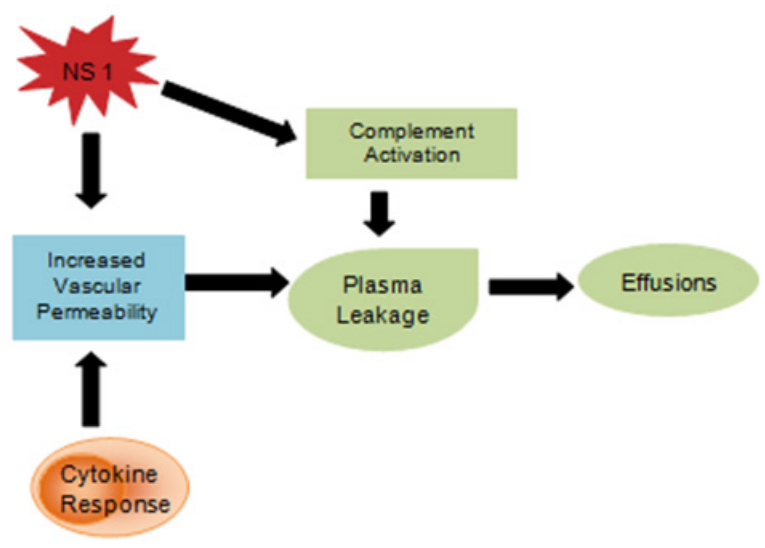

Figure 4 Cytokine immune system response.

During the febrile viremic phase of DHF, the thrombocytopenia and the subsequent manifestations are believed to occur due to bone marrow suppression. As previously mentioned, the suppression of the bone marrow is a result of it being a site of replication for the virus, and specifically attacking hematopoietic progenitor cells, ${ }^{17,18}$ However, immune-mediated destruction of the platelets was also 
suggested by the discovery of virus-antibody complexes found on platelet surfaces of DHF patients.

Uncommon clinical manifestations include acute hepatitis, hepatic failure, acute renal failure, encephalopathy, or cardiomyopathy (acute myocarditis). ${ }^{17}$ A study on fatal DHF in adult looked at causes of fatality other than Dengue shock syndrome (DSS) in patients having severe dengue and concluded that hypothermia, leukocytosis, and bandemia could be trigger signs of severe dengue. The study further advice clinicians to be conscious of possible development of massive GI bleeding, especially in patients having early altered consciousness, profound thrombocytopenia, prolonged PT and/or leukocytosis. ${ }^{25}$

\section{Diagnosis, treatment, and management}

Early and accurate diagnosis is critical for proper clinical care. There are many methods in which the diagnosis of dengue can be made. Diagnosis can be accomplished through the isolation of the virus, the viral nucleic acid, antibodies and antigens, or a combination of all of these techniques. Detection of dengue virus by culture is the definitive diagnostic test, but practical consideration limits its use. Detection of viral RNA is faster but contamination can lead to false positive result. Also, serological test may produce false positive result due to cross reactive antibodies. ${ }^{17}$

Acute infections are detected in serum, plasma, and circulating blood cells during the early convalescent phase, which is the initial 5-8 days of symptoms. At this time, the primary viral load may be as high as $10^{3}$ RNA particles $/ \mathrm{ml}$ or greater than $10^{6}$ RNA particles/ $\mathrm{ml}$ in secondary infections. ${ }^{26}$ Currently, the combination of the NS1 antigen and antibody tests, as well as molecular methods, such as real time reverse transcription-polymerase chain reaction (RT-PCR), are being used more often for diagnosis as they can deliver quicker results and can be more sensitive. ${ }^{26-28}$ Immunohistochemical analysis and immunofluorescence also confirm the presence of the virus through viral antigens. IgM antibodies are the first to appear within the first 3-5 days of infection. ${ }^{29}$

They are at peak levels 2 weeks after symptoms appear then decline generally to undetectable levels after $30-60$ days. ${ }^{30}$ IgG antibodies, which are more cross-reactive, are detected at low levels after the initial 7-9 days of symptoms, increase slowly up to fourfold, and remain detectable after several months and possibly even life. ${ }^{31}$ The human antigen and antibody response is dependent on the immune status of the host and are present only during primary or secondary infection. There are no specific treatments or antiviral drugs for dengue. The pathophysiology of dengue increases vascular permeability, which leads to a significant loss of plasma from the intravascular compartment into extra vascular space.

This loss of fluid can lead to shock and without proper correction will lead to hypoxia, metabolic acidosis, and eventually death. Therefore, primary management requires inpatient supportive care with fluid and electrolyte balance. The packed cell volume should be monitored on the hour every 4-6 hours. Significant plasma loss is defined as having a $>20 \%$ increase in packed cell volume or haematocrit. Once a patient reaches these levels, rapid and aggressive volume replacement is necessary. ${ }^{17,32}$

The infusion fluid replacement rate is dependent on the packed cell volume and the urine output. There are multiple fluids used for plasma loss replacement including physiological saline, Ringer's lactate or Ringer's acetate, diluted 5\% glucose solution, plasma or plasma substitutes such as dextrin 40 and fresh whole blood. ${ }^{33}$ In addition to intravenous fluid replacement, electrolytes can be introduced orally as it will relieve some of the thirst and dehydration from the fever. Supportive analgesics such as acetaminophen can be used to treat fever and other symptoms. NSAIDs and aspirin should be avoided as they might precipitate Reye's syndrome.

Upon recovery of DHF patients must meet the following criteria before hospital discharge: visible clinical improvement, afebrile without the use of antipyretics for $>24$ hours, increased appetite, stable hematocrit, adequate urine output, three days post recovery from severe metabolic acidosis, Platelet count $>50,000 / \mathrm{mm}^{3}$, and no respiratory distress from pleural effusion or Ascites. ${ }^{17,32}$

\section{Prevention and eradication of aedes aegypti}

Aedes aegypti, the most prevalent vector of the dengue virus, is the main target of surveillance and control activities. Other vectors, such as Aedes albopictus, can produce dengue fever, but surveillance and control activities for different vectors should only be considered if there is reliable evidence that those vectors played an epidemiologically significant role in the transmission of dengue infections. ${ }^{34}$ The first initiative to eliminate Aedes aegyptiwas in Cuba in 1901. Fumigation and elimination of the mosquito foci by destroying abandoned containers were the first steps to eradicating the vector. ${ }^{35}$ After outbreaks of yellow fever occurred in Brazil in 1928 and 1932, it was evident that complete protection of urban populations depended on absolute eradication of the Aedes aegyptimosquito; this led to the approval by Pan American Health Organization (PAHO) of the Continental Aedes aegyptieradication plan in 1947 to fight urban yellow fever. ${ }^{35}$ Since 1947, the Pan American Sanitary Bureau (PASB) intensely promoted campaigns in all affected countries, and their success was demonstrated by 1962, when 18 continental countries and a number of Caribbean islands had achieved eradication. As a result of these efforts, the American DENV-2 genotype $\mathrm{V}$ is the only dengue virus that remains today. ${ }^{35}$

Despite the efforts to eradicate the vector, Aedes aegypti was not eradicated in Cuba, the Unites States, Venezuela, and several Caribbean countries. ${ }^{35}$ Decades of unprecedented human efforts to eradicate Aedes aegyptifell apart very rapidly and quickly led to infestation. Lack of surveillance and deterioration of eradication program structure, insufficient environmental sanitation, and insufficient community participation were some of the factors that led to the decline in efforts to eradicate the vector. ${ }^{35}$ As of today, there is no vaccine available to prevent dengue infections. ${ }^{36}$ Takeda Vaccines Inc. is developing a live, attenuated candidate vaccine known as tetravalent dengue vaccine (TDV) which is made up of an attenuated DENV-2 strain (TDV-2) and three chimeric viruses containing the prM and E protein genes of DENV-1, -3 and -4 which were expressed in the context of the attenuated TDV-2 genome backbone (TDV-1, TDV-3, and TDV-4, respectively). The vaccine has been shown to be well tolerated and immunogenic in a Phase 2 clinical trial in dengue endemic countries among adults and children.

The vaccine is on phase 3 stage of development. ${ }^{37}$ In addition, Sanofi Pasteur has developed a recombinant, live-attenuated, tetravalent dengue vaccine (CYD-TDV) that showed consistent safety and efficacy in $\geq 9$ year individuals in endemic countries; it is in the late-stage of development. ${ }^{38}$ Dengvaxia (CYD-TDV) by Sanofi Pasteur was first registered in Mexico in December, 2015 and has been evaluated as a 3 -dose series on a 0/6/12 month schedule in Phase III clinical studies. ${ }^{6}$ There are approximately five additional vaccine candidates under evaluation in clinical trials. ${ }^{6}$ Travelers to dengue-endemic areas are at risk of contracting dengue; thus for prevention, they should be encouraged to select accommodations with 
well-screened windows and doors, use insect repellent, wear proper clothing, and empty and cover any standing water that can possibly become mosquito-breeding sites. ${ }^{39}$

\section{Current global situation}

Currently, there are expectations that the incidence of dengue will continue to rise due many factors including socioeconomic status, continuous climate change, travels, and globalization. Vaccines are still under development and no specific antiviral therapy currently exists to address the growing threat of dengue ${ }^{40}$ The global estimate of the incidence of dengue is between 50 to 200 million. However, the use of cartographic approaches recently suggests that the number of the incidence is closer to 400 million. ${ }^{41}$ In 2012 , the WHO classified dengue as one of the vital mosquito borne viral disease. ${ }^{42}$ This classification is justified by the significant geographical spread of the virus along with its vector to areas that were not previously affected (Figure 5)

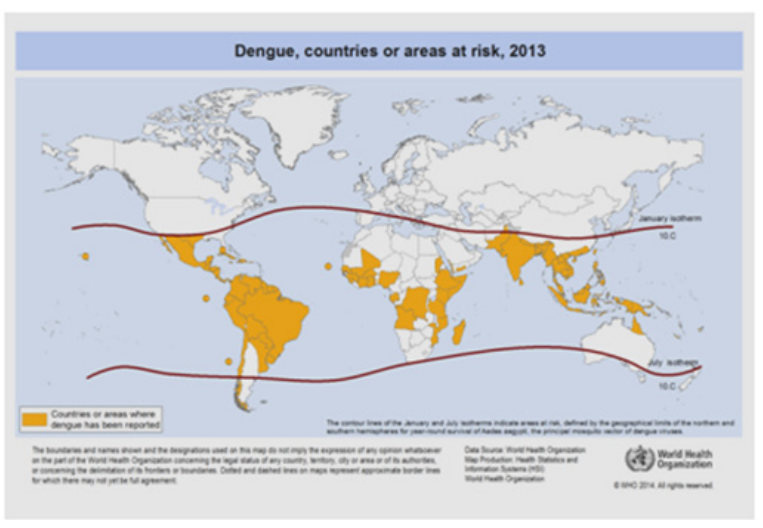

Figure 5 Countries or areas of the world where dengue was reported in 2013 , as per data collected by the World Health Organization. ${ }^{41}$

It is also supported by the cost the disease brings along with it Comparing the burden of dengue and other communicable disease in affected region, dengue causes far more human suffering and is more of a burden than the other vector borne diseases. ${ }^{42}$ According to the WHO, dengue is the leading cause of hospitalization and death in children in Southeast Asia. ${ }^{43}$ A study by Hammond et al. reported that the majority of the morbidity and mortality related to this disease is associated with children rather than any other age groups. Infants between 4-9 months of age and children between the ages of 5-9 years carry the highest burden of dengue (Figure 6). The severity of the disease in these children is often due to secondary DENV infection. ${ }^{44}$ An ecological study carried out by Diaz-Quijano and Waldman in Latin America and the Caribbean found that the determinants of the dengue mortality rate in these populations included the length of recognized endemicity, population density, and rainfall. ${ }^{45}$

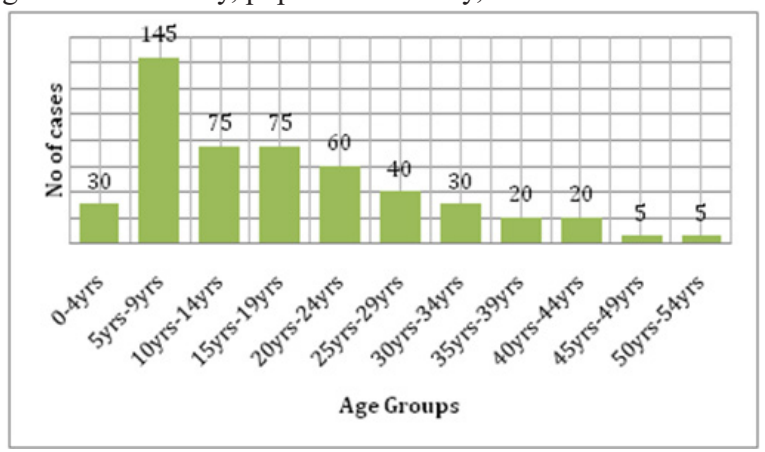

Figure 6 Age distribution of study cohort in León by age group. ${ }^{44}$
In addition to the impact of morbidity and mortality, dengue virus carries a high financial burden to affected regions, many of which are ill equipped to bear these costs. In 2010, the financial burden of the dengue virus was estimated to be $\$ 2.1$ billion per year on average in the Americas (in 2010 US dollars), with a range of \$1-4 billion in sensitivity analyses and substantial year-to-year variation. ${ }^{46}$ According to the WHO, dengue is now seen as a worldwide concern with the Asian-Pacific fostering the highest (75\%) global population with exposure to the dengue virus. ${ }^{40}$ The Western Pacific also has experienced a continuous increase in the number of reported cases of DF over the past decade. ${ }^{47}$

Even with the absence of transmission of DF in the middle 20th century, there is now the presence of an increased endemic of indigenous DF in the Americas. In the Americas alone, in 2010, there were over 1.6 million reported cases of dengue and 49,000 of these being severe dengue. ${ }^{40}$ In Africa, outbreaks of DF are increasing in size and frequency but are not being consistently reported to the WHO. Between 1960 and 2010, sporadic cases were reported by 22 African countries as suggested by available outbreak data. ${ }^{40}$ In Europe, no epidemics of DF have been reported since an outbreak in Greece in 1928. There are now threats of the outbreak in Europe, which has been attributed to Aedes albopictus due to travel from countries where the disease is endemic. It is estimated that there will be an increase in incidence of the disease in the years to come owing to multifactorial risk factors. ${ }^{40}$

\section{Conclusion}

Since dengue is no longer confined to the tropics, it is currently the most prevalent mosquito-borne viral disease among humans and is considered a major international public health concern today. A worsening case of dengue leads to an associated hemorrhagic fever, resulting in several hospitalizations and deaths among the pediatric population. Additionally, socioeconomic status, globalization, climate and temperature changes are highly involved in the spread of this viral illness and many scientists and healthcare professionals believe that dengue will continue to be on the rise especially when influenced by these factors.

\section{Conflicts of interest}

None.

\section{References}

1. World Health Organization. Impact of dengue. 2015.

2. Malavige GN, Fernando S, Fernando DJ, et al. Dengue viral infections. Postgrad Med J. 2004;80(948):588-601.

3. Gubler DJ. Dengue and dengue hemorrhagic fever. Clin Microbiol Rev 1998;11(3):480-496.

4. Gubler DJ, Clark GG. Dengue/dengue hemorrhagic fever: the emergence of a global health problem. Emerg Infect Dis. 1995;1(2):55-57.

5. Stephenson JR. Understanding dengue pathogenesis: Implications for vaccine design. Bull World Health Organ. 2005;83(4):308-314

6. World Health Organization. Dengue and severe dengue: Fact Sheet No 117, Geneva. 2012.

7. Centers for Disease Control and Prevention. CDC - EpidemiologyDengue. 2014.

8. Kraemer M, Sinka ME, Duda KA, et al. The global distribution of the arbovirus vectors Aedes aegypti and Aedes albopictus |eLife. 2015;4:e08347. 
9. Lambrechts L, Scott TW, Gubler DJ. Consequences of the expanding global distribution of Aedes albopictus for dengue virus transmission. PLoS neglected tropical diseases. 2010;4(5):e646.

10. Guzman MG, Halstead SB, Artsob H, et al. Dengue: a continuing global threat. Nat Rev Microbiol. 2010;8(12 Suppl):S7-16.

11. Hales S, De Wet N, Maindonald J, et al. Potential effect of population and climate changes on global distribution of dengue fever: an empirical model. The Lancet. 2002;360(9336):830-834.

12. Samuel PP, Thenmozhi V, Nagaraj J, et al. Dengue vectors prevalence and the related risk factors involved in the transmission of dengue in Thiruvananthapuram district, Kerala, South India. J Vector Borne Dis. 2014;51(4):313-319.

13. Cory W Morin, Andrew C Comrie, Kacey Ernst. Climate and Dengue Transmission: Evidence and Implications. Environmental health perspectives. $2013 ; 121(11-12): 1-9$.

14. Gubler D. Epidemic dengue/dengue hemorrhagic fever as a public health, social and economic problem in the 21 st century. Trends Microbiol. 2002;10(2):100-103.

15. Martina BE, Koraka P, Osterhaus AD. Dengue Virus Pathogenesis: an Integrated View. Clin Microbiol Rev. 2009;22(4):564-81.

16. Tsai TT, Chuang YJ, Lin YS, et al. An emerging role for the antiinflammatory cytokine interleukin-10 in dengue virus infection. $J$ Biomed Sci. 2013;20(40):1-9.

17. World Health Organization. Dengue Haemorrhagic Fever: Diagnosis, Treatment, Prevention and Control, (2nd edn). Geneva: World Health Organization, USA. 1998

18. Sellahewa KH. Pathogenesis of dengue haemorrhagic fever and its impact on case management. ISRN Infectious Diseases. 2013:1-6.

19. Chuang YC, Wang SY, Lin YS, et al. Re-evaluation of the pathogenic roles of nonstructural protein 1 and its antibodies during dengue virus infection. J Biomed Sci. 2013;20(42):1-7.

20. Avirutnan P, Matangkasombut P. Infectious disease: unmasking the role of mast cells in dengue. Elife. 2013;(2):e00767.

21. Kolitha HS. Pathogenesis of dengue haemorrhagic fever and its impact on case management. ISRN Infectious Diseases, 2013:1-6.

22. Wahid SF, Sanusi S, Zawawi MM, et al. A comparison of the pattern of liver involvement in dengue hemorrhagic fever with classic dengue fever Southeast Asian Journal of Tropical Medicine and Public Health. 2000;31(2):259-263.

23. San Martín JL, Brathwaite O, Zambrano B, et al. The epidemiology of dengue in the Americas over the last three decades: a worrisome reality. Am J Trop Med Hyg. 2010;82(1):128-35.

24. Hause AM, Perez-Padilla J, Horiuchi K, et al. Epidemiology of Dengue among Children Aged< 18 Months-Puerto Rico, 1999-2011. Am J Trop Med Hyg. 2016;94(2):404-408.

25. Lee K, Liu JW, Yang KD. Fatal dengue hemorrhagic fever in adults: emphasizing the evolutionary pre-fatal clinical and laboratory manifestations. PLoS Negl Trop Dis. 2012;6(2):e1532.

26. Johnson BW, Russell BJ, Lanciotti RS. Serotype-specific detection of dengue viruses in a fourplex real-time reverse transcriptase PCR assay. J Clin Microbiol. 2005;43(10):4977-4983.

27. Kassim FM, Izati MN, TgRogayah TA, et al. Use of dengue NS1 antigen for early diagnosis of dengue virus infection. Southeast Asian J Trop Med Public Health. 2011;42(3):562-569.
28. Gowri Sankar S, Dhananjeyan KJ, Paramasivan R, et al. Evaluation and use of NS1 IgM antibody detection for acute dengue virus diagnosis: report from an outbreak investigation. Clin Microbiol Infect. 2012;18(1):E8-10.

29. Martin DA, Muth DA, Brown T, et al. Standardization of immunoglobulin M capture enzyme-linked immunosorbent assays for routine diagnosis of arboviral infections. J Clin Microbiol. 2000;38(5):1823-1826.

30. World Health Organization. Dengue: Guidelines for Diagnosis, Treatment, Prevention and Control. (New edn)., Geneva: 4). Laboratory Diagnosis and Diagnostic Tests, Switzerland, pp. 2009;3-160.

31. Innis BL, Nisalak A, Nimmannitya S, et al. An enzyme-linked immunosorbent assay to characterize dengue infections where dengue and Japanese encephalitis co-circulate. Am J Trop Med Hyg. 1989;40(4):418-427.

32. Gurugama P, Garg P, Perera J, et al. Dengue viral infections. Indian journal of dermatology. 2010;55(1):68-78.

33. World Health Organization. Chapter 3 Treatment. Dengue haemorrhagic fever: Diagnosis, treatment, prevention, and control. (2nd edn). Geneva, Switzerland, p. 1997.24-33.

34. Carrington LB, Simmons CP. Human to mosquito transmission of dengue viruses. Front Immunol. 2014;5:1-290.

35. Dick OB, San Martín JL, Montoya RH, et al. The history of dengue outbreaks in the Americas. Am J Trop Med Hyg. 2012;87(4):584-593.

36. Thomas SJ, Endy TP. Vaccines for the prevention of dengue: development update. Human vaccines. 2015;7(6):674-684.

37. Osorio JE, Partidos CD, Wallace D, et al. Development of a recombinant, chimeric tetravalent dengue vaccine candidate. Vaccine. 2015;33(50):7112-7120

38. Guy B, Briand O, Lang J, et al. Development of the Sanofi Pasteur tetravalent dengue vaccine: One more step forward. Vaccine. 2015;33(50):7100-7111.

39. Tomashek KM, Margolis HS. Dengue: a potential transfusiontransmitted disease. Transfusion. 2011;51(8):1654-1660.

40. Murray NE, Quam MB, Wilder-Smith A. Epidemiology of dengue: past, present and future prospects. Clin Epidemiol. 2013;5:299-309.

41. World Health Organization. Dengue, countries or areas at risk, 2011 Geneva, Switzerland. 2011.

42. World Health Organization. Global Strategy for Dengue Prevention and Control, 2012-2020. Geneva, Switzerland. 2012.

43. World Health Organization. Comprehensive Guidelines for Prevention and Control of Dengue and Dengue Haemorrhagic Fever. WHO Regional Office for South-East Asia. Revised and Expanded Edition. New Delhi: World Health Organisation South East Asia Regional Office, India, pp. 2011;1-212.

44. Hammond SN, Balmaseda A, Perez L, et al. Differences in dengue severity in infants, children, and adults in a 3-year hospital-based study in Nicaragua. Am J Trop Med Hyg. 2005;73(6):1063-1070.

45. Díaz-Quijano FA, Waldman EA. Factors associated with dengue mortality in Latin America and the Caribbean, 1995-2009: an ecological study. Am J Trop Med Hyg. 2012;86(2):328-334.

46. Shepard DS, Coudeville L, Halasa YA, et al. Economic impact of dengue illness in the Americas. Am J Trop Med Hyg. 2011;84(2):200-207.

47. Arima Y, Matsui T. Epidemiologic update of dengue in the Western Pacific Region, 2010. Western Pac Surveill Response J. 2011;2(2):4-8. 